nombreux domaines. Vu l'importance de la coopération de l'industrie dans la fabrication et la diffusion des systèmes de convertisseurs d'images à usage astronomique, l'Union Astronomique Internationale peut et devrait user de son influence et prestige dans ce problème. Il est donc souhaitable que l'importance des convertisseurs d'images et leurs applications à l'astronomie soient reconnues et trouvent une place dans l'activité de l'Union.

En terms plus précis, le rôle initial d'une sous-commission concernant les convertisseurs d'images photoélectriques pourrait être: l'encouragement d'une fabrication commerciale des élements dont dépend la généralisation de ces méthodes, la normalisation des charactéristiques d'adaptation aux instruments astronomiques et aux spécifications des appareils commerciaux, l'obtention des facilités vis-à-vis des brevets et licenses en ce qui concerne les applications astronomiques, et l'encouragement des recherches ultérieures.

Nous souhaiterions donc que l'Union veuille bien approuver l'établissement d'une sous-commission concernant les Convertisseurs d'Images au sein de la Commission 9 (Instruments). Le président par interim a marqué son accord à ce sujet. Nous suggérons que la sous-commission concernant les Convertisseurs d'Images comprenne les membres suivants, qui travaillent dans ce domaine: BAum (président), Duchesne, Fellgett, J. S. Hall, Hiltner, Krassovsky, Lallemand, Leighton, McGee, Morton, and A. G. Wilson.

\title{
Commission Io
}

r. La Commission recommande la mise au point précise, à l'occasion de l'Année Géophysique Internationale, de l'étude détaillée en commun de l'évolution des taches solaires, sous la direction de l'Observatoire fédéral de Zurich, conformément à la résolution adoptée par la septième Assemblée générale de l’U.A.I. en I948.

2. La Commission recommande que la subvention annuelle de $x 000$ francs-or, accordée précédemment à l'Observatoire de Zurich pour la publication des Cartes héliographiques de la Photosphère, soit renouvelée pour la période qui s'étendra jusqu'à la prochaine Assemblée générale.

3. La Commission recommande qu'une subvention annuelle de I500 dollars soit accordée à l'Observatoire de Zurich pour la publication du Quarterly Bulletin on Solar Activity, pendant la période qui s'écoulera jusqu'à la prochaine Assemblée générale.

\section{Commission II}

I. La Commission recommande que la subvention annuelle de 2700 francs-or, accordée depuis I925 par l'U.A.I. à l'Observatoire de Meudon pour la publication des Cartes synoptiques de la Chromosphère solaire, soit renouvelée pour la période qui s'écoulera jusqu'à la prochaine Assemblée générale.

2. La Commission recommande que le nouveau mode de classification des éruptions solaires tel qu'il est présenté dans la Section 3 (page I53 de ce volume), soit adopté à partir du premier janvier $195^{6}$.

\section{Commission $\mathbf{I 2}$}

I. La Commission recommande instamment à tous les laboratoires de physique et tous les laboratoires d'Astrophysique convenablement outillés la détermination des 'forces d'oscillateur' (valeurs de $f$ ) et autres constantes astrophysiques dont les astronomes ont un besoin urgent.

2. La Commission recommande instamment l'affectation d'un crédit de 2000 dollars à la publication des Tables spectroscopiques solaires (Solar Spectroscopic Tables), dont on prévoit l'achèvement avant la prochaine Assemblée de l'U.A.I. en 1958.

Ces tables sont d'une importance capitale pour tous les travaux de spectroscopie solaire et stellaire. 